\title{
Introducing a New Method for Efficient Visualization of Complex Shape 3D Ultrasonic Phased-Array C-Scans
}

\author{
Carmelo Mineo, Rahul Summan, Jonathan Riise, Charles N. MacLeod, S. Gareth Pierce \\ Centre for Ultrasonic Engineering, Department of Electronic and Electrical Engineering, University of Strathclyde \\ Glasgow, United Kingdom \\ carmelo.mineo@strath.ac.uk
}

\begin{abstract}
Automated robotic inspection systems allow the collection of large data volumes, compared to existing inspection systems. To maximize the throughput associated with the nondestructive evaluation phase, it is crucial that the reconstructed inspection data sets are generated and examined rapidly without a loss of detail. Data analysis often becomes the bottleneck of automated inspections. Therefore, new data visualization tools, suitable to screen the NDT information obtained through robotic systems, are urgently required. This paper presents a new approach, for the generation of three-dimensional ultrasonic $\mathrm{C}$ scans of large and complex parts, suitable for application to high data throughput ultrasonic phased array inspection. This approach produces 3D $\mathrm{C}$-scan presented as colored tessellated surfaces and the approach works efficiently on challenging geometry, with concave and convex regions. Qualitative and quantitative results show that the approach runs up to 500 times faster than other $\mathbf{C}$-scan visualization techniques.
\end{abstract}

Keywords - C-scans; Phased arrays; Data visualisation; Robotics; Automated systems

\section{INTRODUCTION}

Ultrasonic testing is one of the most widely used techniques in non-destructive testing and evaluation (NDT). To cope with future demand projections (e.g. in the aerospace sector) and overcome the bottlenecks of safety critical NDT inspections, new automated scanning systems have been developed by a variety of research and development teams [1,2]. Producing reliable automated solutions has become an industry priority to drive down inspection times and ensure repeatability. Applications of six-axis robotic arms in the NDT field have been published during the last few years and there is a growing interest in using such automation solutions [3, 4].

Ultrasonic data can be collected and displayed in a number of different formats. Notoriously, the three common formats are known as A-scan, B-scan and C-scan presentations; the latter provide a feature map view of the position and size of specimen features. The plane of the image is parallel to the scan pattern of the transducer. The relative signal amplitude or the time-offlight is displayed as a shade of grey or a colour map tint for each of the positions where data are recorded. The C-scan presentations provide an image of the features of the test piece that reflect and scatter the sound. These presentations have become by far the most common way ultrasonic data are visualized, since they allow screening the full extent of the specimen at once. When scanning highly curved surfaces, traditional C-scans images are typically shown as projections of the scanning trajectory on the X-Y plane. Sizing of the specimen features and evaluation of the true relative distances between

This work is part of the Autonomous Inspection in Manufacturing and Re-Manufacturing (AIMaReM) project, funded by the UK Engineering and Physical Science Research Council (EPSRC). them become challenging, since the three-dimensional nature of the specimen under inspection is lost in a bi-dimensional C-scan presentation. Evaluating the correct 3D distance between indications is crucial in applications where the standards impose limits on the defect density (number of defects per surface unit), besides on the maximum defect size [5].

A possible solution consists of partitioning large scans into small rectangular regions, where approximating the real surface geometry to a plane can be tolerated. Besides producing approximated sizing and measurements, this approach also complicates and slows down the data analysis process. Robotic inspection systems currently under development enable scanning large surfaces (e.g. a full aerospace wing) in a single pass [6]. Given the limitations of the traditional C-scan presentations, researchers have started developing new data analysis software interfaces to facilitate the visualization and the examination of large NDT datasets $[6,7]$. This work presents a new approach developed to revolutionise the visualization of three-dimensional C-scan maps based on a method of index triangulation. This paper explains the mathematical formulation of the new Index Based Triangulation technique. The advantages of the new approach are demonstrated and discussed.

\section{REVIEW OF POSSIBLE VISUALIZATION APPROACHES}

Generating a three-dimensional C-scan map for curved surfaces is not a trivial task. This section presents a review of the visualization approaches available to date. The understanding of the existing methods is necessary to discuss the novelty aspects and the advantages of the new approach presented in this work.

\section{A. Traditional $2 D$ image presentation}

The 2D presentation assumes the A-scans are collected at constant intervals and aligned in a rectangular grid. Therefore, the resulting C-scan image is an $m$-by- $n$ grid of pixels where $m$ is the number of columns and $n$ is the number of rows. The $\mathrm{X}$ and $\mathrm{Y}$ coordinates of each A-scan acquisition location determine the centre of the corresponding pixel in the image. Given that the specimen is scanned at finite resolution and the A-scans are collected at fixed intervals, when a small section of the C-scan image is magnified, the single-coloured square elements that comprise the image (pixels) become visible and the image is said to be pixelated. Fig. 1 shows an ultrasonic scan of a $70 \times 100 \mathrm{~mm}$ steel plate using a KUKA KR5 robotic manipulator. 

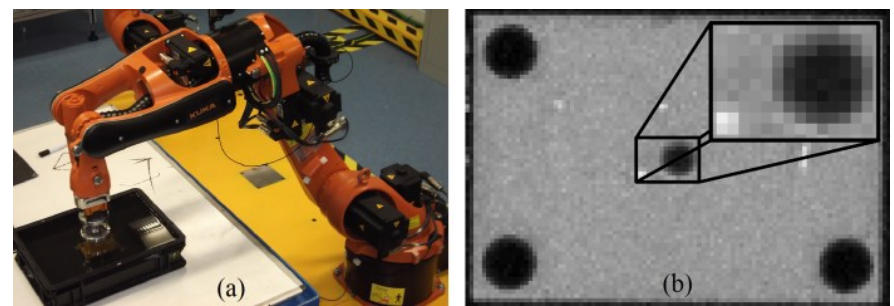

Fig. 1. Sample scanned through a robotic arm (a). 2D C-scan image showing a magnified area, which illustrates the pixelated nature of the data (b).

The plate was scanned through a raster tool-path with the robot stopping at every $1 \mathrm{~mm}$ interval along the $\mathrm{x}$-direction and stepping by $1 \mathrm{~mm}$ in the $y$-direction at the end of each scanning pass. An area of $11 \times 15 \mathrm{~mm}$, magnified 2.5 times, is shown in the top right corner of the resulting traditional 2D C-scan map, (Fig. 1b). It reveals the presence of an $8 \mathrm{~mm}$ diameter flat bottom hole not visible from the top surface of the specimen. The limited resolution of robot joint encoders produces points with $\mathrm{X}$ and $\mathrm{Y}$ coordinates that differ by small deviations from the points on the ideal programmed robot trajectory. Rounding of the coordinates allows presenting the $\mathrm{C}$-scan as an image.

\section{B. Coloured Point Cloud}

An alternative way of displaying $\mathrm{C}$-scan maps is as a point cloud with each point coloured according to the relative signal amplitude or the time-of-flight value originating from gated signal measurements. This visualization method allows using the raw point coordinates obtained directly from the manipulator encoders. Therefore, the robot can manipulate the probe through a continuous motion and A-scans can be acquired at variable intervals, since it is not necessary for the points to be aligned according to a rectangular grid. The coloured point cloud display is intrinsically three-dimensional. However, a point-cloud based $\mathrm{C}$-scan display has some significant drawbacks. Given a certain size to the data points, some of them will overlap if their distance is lower than the average resolution value. The overlap increases when the display is zoomed out. Gaps can appear between adjacent elements that are a bit further from each other. The element separation increases when the C-scan map is magnified. This inconvenience can significantly hinder correct analysis of the inspection data. Fig. 2a shows the point cloud based C-scan map relative to the same specimen mentioned previously. The magnified region shows the unwanted rarefaction of the points.

\section{Tessellated Surfaces}

The problem of overlapping points and rarefaction can be overcome by presenting the $\mathrm{C}$-scan as a tessellated surface rather than a point cloud. Given the $3 \mathrm{D} \mathrm{C}$-scan data points, it is possible to use algorithms able to generate a mesh where each set of three close points become the vertices of one triangle. A triangle is the smallest geometrical entity with a finite surface that can be used to connect three points. In a triangle mesh, the triangles are connected by their common edges or corners. Therefore, a tessellated surface consists in triangles that fill the gaps between the data points. In the visualization of a tessellated surface, the graphics engine does not need to turn the points into graphical objects. Tessellated surfaces are the workhorse of graphical engines. Several visualization algorithms have been optimized for them (e.g. zooming, panning and 3D rotation) [8]. There are two different ways to create a triangular tessellated surface from

This work is part of the Autonomous Inspection in Manufacturing and Re-Manufacturing (AIMaReM) project, funded by the UK Engineering and Physical Science Research Council (EPSRC). a point cloud: using triangulation methods or surface reconstruction methods. Triangulation algorithms use the original points of the input point cloud, using them as the vertices of the mesh triangles. Therefore, the colour given to the face of every triangle is extrapolated from the ultrasonic measurements associated to the triangle vertices. The Delaunay triangulation is the most popular algorithm of this kind [9]. Fig. $2 \mathrm{~b}$ shows the $\mathrm{C}$-scan map plotted through the Delaunay mesh. Surface reconstruction algorithms differ from the triangulation method since they do not use the original points as the vertices of the mesh triangles but compute new points, whose density can vary according to the local curvature of the $3 \mathrm{D}$ geometry. The Poisson's surface reconstruction method is greatly used in several software applications for mesh manipulation and processing, where it is useful to reconstruct the geometry of object scanned through scanning technologies [10]. Fig. 2c shows the C-scan map plotted through the Poisson's reconstructed surface. The surface reconstruction algorithms are not ideal to generate mesh-based $\mathrm{C}$-scan presentations. Wherever the point density is decreased, the sizing accuracy with which is possible to evaluate the $\mathrm{C}$-scan indications is adversely affected.

\section{NEW INDEX BASED TRIANGULATION (IBT) APPROACH}

An intrinsic characteristic of the A-scans collected to create a $\mathrm{C}$-scan map is the sequential way they are acquired along the scanning trajectory. The ultrasonic probe is moved along the predefined trajectory to cover the whole extent of the surface of interest and the A-scans are collected at regular intervals. The level of orderliness is even higher when the ultrasonic inspection is carried out through a phased array probe. The ordered spatial distribution of the A-scans locations, obtained through ultrasonic phased array probes, inspired the development of an efficient triangulation algorithm targeted to such structured point distribution. Given the number of A-scans $(N)$ contained in a Bscan frame and the total number of frames $(F)$, it is possible to create a table with the indices of the points to link, in order to create a neat and efficient triangulation. This approach is hereafter named as the Index Based Triangulation (IBT) algorithm.
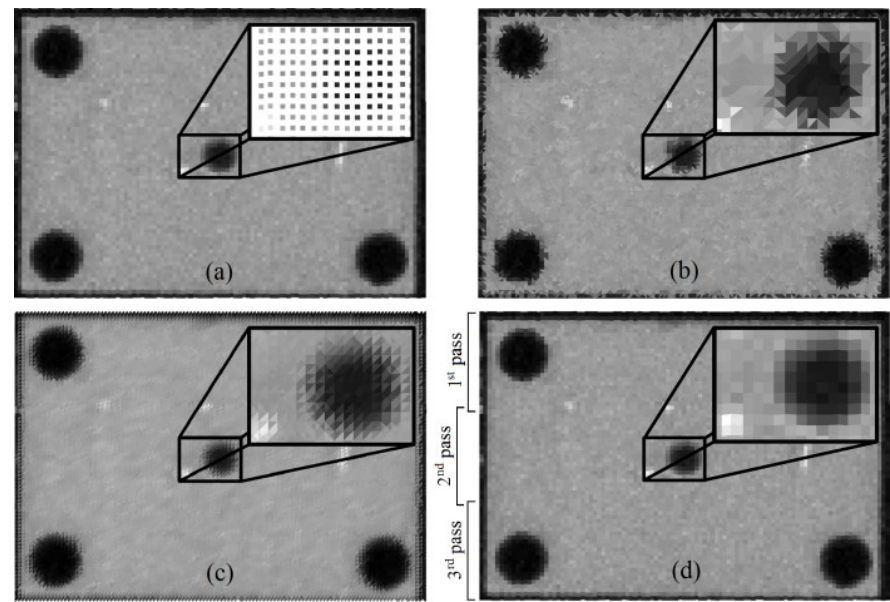

Fig. 2. C-scan plotted as point cloud (a), Delaunay triangulation (b), Poisson surface reconstruction (c) and IBT mesh (d). 
Assigning increasing indices to the points according to the electronic scanning direction within each frame and the mechanical scanning direction from frame 1 to frame $F$, the point indices span from 1 to $[F \cdot N]$. The indices of the points in the $j^{\text {th }}$ frame go from $[(j-1) N+1]$ to $[j N]$. The index of the point relative to the $i^{\text {th }}$ pulse in the $j^{\text {th }}$ frame is $[(j-1) N+i]$. Fig. 3 shows the triangulation of the $\mathrm{C}$-scan frames according to the IBT method. The numbering of the points and triangles reproduce the relative indices. The points of the $j^{\text {th }}$ frame form $2(N-1)$ triangles with the points of the $(j+1)^{\text {th }}$ frame. From the first to the second-last point of the $j^{\text {th }}$ frame, each point is the vertex of two triangles. For example, the first and second frame produce the triangulation given in Fig. 3. Each row of the table specifies a triangle, defined by indices with respect to the points. The triangulation between the $j^{\text {th }}$ and the $(j+1)^{\text {th }}$ frame is given by a copy of Table 1 , with all point indices incremented by ( $\mathrm{j}$ 1)N. Therefore, the full triangulation for the $\mathrm{C}$-scan can be expressed by a matrix $(\mathrm{T})$ representing the set of triangles that make up the triangulation.

The matrix is generated by appending $(F-1)$ copies of the $2(\mathrm{~N}-1)$ triangles in Table 1 , were the values of each copy is incremented by the integer number $[(j-1) N]$, with $j$ varying between 1 and $(F-1)$. The triangulation produces a meshed stripe whose length depends on the distance travelled by the probe and the width $(w)$ is equal to $w=(N-1) \cdot p$, where $\mathrm{p}$ is the pitch of the phased array probe.

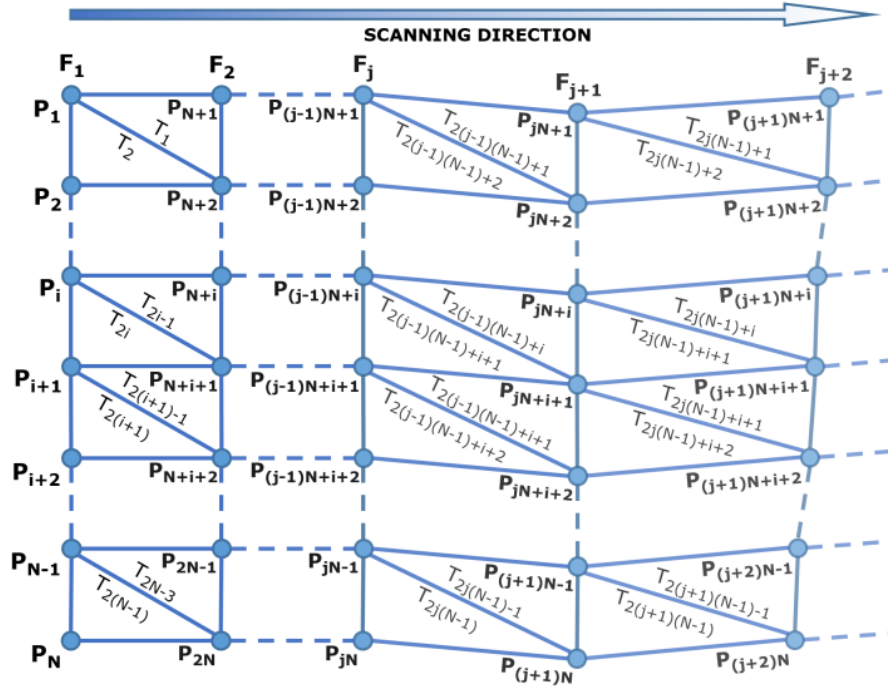

Fig. 3. Indexes for the Index Based Triangulation (IBT) algorithm.

TABLE I.

Triangulation OF THE FiRst Two Frames.

\begin{tabular}{|l|l|l|l|}
\hline Tringles & Vertex 1 index & Vertex 2 index & Vertex 3 index \\
\hline $\mathrm{T}_{1}$ & $\mathrm{P}_{1}$ & $\mathrm{P}_{\mathrm{N}+1}$ & $\mathrm{P}_{\mathrm{N}+2}$ \\
\hline $\mathrm{T}_{2}$ & $\mathrm{P}_{1}$ & $\mathrm{P}_{\mathrm{N}+2}$ & $\mathrm{P}_{2}$ \\
\hline \hline $\mathrm{T}_{2 \mathrm{i}-1}$ & $\mathrm{P}_{\mathrm{i}}$ & $\mathrm{P}_{\mathrm{N}+\mathrm{i}}$ & $\mathrm{P}_{\mathrm{N}+\mathrm{i}+1}$ \\
\hline $\mathrm{T}_{2 \mathrm{i}}$ & $\mathrm{P}_{\mathrm{i}}$ & $\mathrm{P}_{\mathrm{N}+\mathrm{i}+1}$ & $\mathrm{P}_{\mathrm{i}+1}$ \\
\hline $\mathrm{T}_{2(\mathrm{i}+1)-1}$ & $\mathrm{P}_{\mathrm{i}+1}$ & $\mathrm{P}_{\mathrm{N}+\mathrm{i}+1}$ & $\mathrm{P}_{\mathrm{N}+\mathrm{i}+2}$ \\
\hline $\mathrm{T}_{2(\mathrm{i}+1)}$ & $\mathrm{P}_{\mathrm{i}+1}$ & $\mathrm{P}_{\mathrm{N}+\mathrm{i}+2}$ & $\mathrm{P}_{\mathrm{i}+2}$ \\
\hline \hline & & & \\
\hline $\mathrm{T}_{2 \mathrm{~N}-3}$ & $\mathrm{P}_{\mathrm{N}-1}$ & $\mathrm{P}_{2 \mathrm{~N}-1}$ & $\mathrm{P}_{2 \mathrm{~N}}$ \\
\hline $\mathrm{T}_{2(\mathrm{~N}-1)}$ & $\mathrm{P}_{\mathrm{N}-1}$ & $\mathrm{P}_{2 \mathrm{~N}}$ & $\mathrm{P}_{\mathrm{N}}$ \\
\hline
\end{tabular}

This work is part of the Autonomous Inspection in Manufacturing and Re-Manufacturing (AIMaReM) project, funded by the UK Engineering and Physical Science Research Council (EPSRC).
There are situations where, for a correct visualization of the Cscan, two consecutive frames must not be linked by triangles. This is the case when the acquisition of frames is paused during the movement over an area without NDT interest (e.g. a hole in the specimen) or during the step movement at the end of each pass. If $\boldsymbol{J}$ is the array of indices of the last frames acquired before such pauses, the unwanted triangles can be removed from $\boldsymbol{T}$, by deleting the matrix rows between $[2(J-1)(N-1)+1]$ and $[2 \boldsymbol{J}(\boldsymbol{N}-1)]$. Assuming $n_{J}$ is the number of frame indices contained in $\boldsymbol{J}$, the full triangulation matrix will have size of $n$ Tri-by-3, with $n$ Tri equal to $n$ Tr $i=n_{T}-2 n_{J}(N-1)$, where $n_{T}$ is the total number of triangles initially created by the described index based triangulation, $n_{T}=2 \cdot(F-1) \cdot(N-$ 1).

The IBT based C-scan can be plotted in MATLAB ${ }^{\circledR}$, through the function: $\operatorname{trisurf}(\boldsymbol{T}, X, Y, Z, C)$. The function displays the triangles defined in the nTri-by- 3 triangulation matrix $\boldsymbol{T}$ as a surface. As described earlier, each row of $\boldsymbol{T}$ contains indexes into the $\mathrm{X}, \mathrm{Y}$, and $\mathrm{Z}$ vertex vectors to define a single triangular face. The colour of the triangles is defined by the vector $C$. Since the IBT approach triangulates the original $C$ scan points, the vector $C$ is the array of the original ultrasonic measurements collected from the gated A-scans. The points of the steel plate sample C-scan were originally acquired with a single ultrasonic transducer manipulated by a robotic arm through discreet point-to-point movements. The order of the points was rearranged to simulate the point cloud that would be acquired by a phased array probe with $N_{P}=32, N_{S}=8, s=1$ and $p=1 \mathrm{~mm}$. With such parameters, the height of the plate is covered by three inspection raster scan passes with width $(w)$ equal to $24 \mathrm{~mm}$, overlapping by $1 \mathrm{~mm}$. Fig. $2 \mathrm{~d}$ shows the $\mathrm{C}$-scan map obtained through the application of the IBT algorithm to such rearranged point cloud distribution. The magnification of the central areas illustrates how the IBT C-scan looks similar to the pixelated image-based $\mathrm{C}$-scan in Fig. 1b, with the advantage that the triangular mesh uses the original A-scan locations as triangle vertices.

\section{QUANTITATIVE PERFORMANCE RESULTS}

Two additional C-scan datasets were acquired through a robotic phased array inspection system. All C-scans were used to compare the computational efficiency of the IBT method with the Delaunay triangulation and the Poisson's algorithm quantitatively. The second obtained C-scan contains 793,572 Ascan points and the third $\mathrm{C}$-scan over 2.7 million points. The three algorithms were tested, using a computing machine based on an Intel ${ }^{\circledR}$ Core(TM) i7-6820HQ CPU (2.70GHz). The benchmarking was carried out through MATLAB ${ }^{\circledR}$ 2016a, running on the Windows 10 64-bit operating system. The inbuilt MATLAB ${ }^{\circledR}$ function, $\boldsymbol{T}=\operatorname{delaunay}(X, Y)$, was used to test the Delaunay triangulation. This function produces good results only when the input points are distributed on a surface that can be mathematically described by a surjective function in the X-Y domain. The 3D version of Delaunay triangulation, $\boldsymbol{T}=$ delaunay $(X, Y, Z)$, is able to operate with non-surjective surfaced, as long as they are closed surfaces and threedimensionally convex. These constraints make the 3D Delaunay function of no utility in our case, so the 2D Delaunay function was used. The Poisson's surface reconstruction algorithm of M. 
Kazhdan [10], coded in $\mathrm{C}++$ programming language and compiled as a MATLAB ${ }^{\circledR}$ MEX-File, was used to test the Poisson's method. The IBT approach was implemented in a MATLAB $\AA$ function by the authors of the present work. Table 2 reports the benchmarking results.

TABle II. Performance of Delaunay triangulation, Poisson's SURFACE RECONSTRUCTION AND IBT METHOD, FOR THREE INPUT DATASETS.

\begin{tabular}{|l|l|l|l|l|}
\hline & & c-Scan \#1 & c-Scan \#2 & c-Scan \#3 \\
\hline \multirow{2}{*}{$\begin{array}{l}\text { Delaunay } \\
\text { triangulation }\end{array}$} & Time [ms] & 20.2 & 3109.1 & 12591.2 \\
\cline { 2 - 5 } $\begin{array}{l}\text { Poisson's } \\
\text { surface }\end{array}$ & Triangles & 15,085 & $1,586,848$ & $5,502,157$ \\
\cline { 2 - 5 } $\begin{array}{l}\text { IBT } \\
\text { algorithm }\end{array}$ & Triangles & 2905.0 & 43481.9 & 82653.3 \\
\cline { 2 - 5 } & Time [ms] & 0.3 & $2,534,808$ & $5,876,655$ \\
\hline \multirow{4}{*}{} & Triangles & 14,400 & 56.3 & 179.2 \\
\hline
\end{tabular}

The Poisson's formulation generated the highest number of triangles for all datasets. Moreover, the new vertex points generated by the Poisson's method required interpolating of the ultrasonic measurements associated to the original points. Besides altering the original spatial sampling resolution of the original point cloud, the surface reconstruction method revealed to be the most time-consuming approach, requiring over 80 seconds for the largest dataset. The Delaunay triangulation is faster than the surface reconstruction and produces a smaller number of triangles. The method required 12.6 seconds for the largest dataset. The new IBT approach generated the smallest number of triangles for all datasets. The method creates simple meshed stripes of triangulated frame points. The IBT algorithm was by far the fastest, with computation times of $0.3 \mathrm{~ms}, 56.3 \mathrm{~ms}$ and $179.2 \mathrm{~ms}$, respectively for the smallest, medium and largest dataset. The IBT method shows enhanced performance because the triangulation algorithm requires only the construction of a matrix of indices. The computer CPU performs simple algebraic operations on integers, rather than double precision numbers.

\section{DISCUSSION OF ADDITIONAL BENEFITS}

The colour and reflectivity that graphic engines assign to each triangle is strongly affected by the normal to the triangle surface. The normal of a triangle is computed by taking the cross product of two of its edges. Reversing the order of the triangle vertices flips the direction of the triangle normal. The Delaunay triangulation and the Poisson's surface reconstruction are unable to maintain a consistent direction in the linkage of the triangle vertices. This has an undesired effect on the colouring of the resulting $\mathrm{C}$-scan maps (see Fig. $2 \mathrm{~b}$ and Fig. 2c). The IBT algorithm ensures that all triangle vertices are connected clockwise, producing coherent normals and colouring. Surfaces can have convex and concave areas. The Delaunay triangulation is only suitable for triangulating convex surfaces. This is an enormous inconvenience in the generation of tessellated surfaces for C-scan presentations. A similar problem is found with the Poisson's surface reconstruction method. In this case, the generation of unwanted triangles arises because the Poisson's algorithm does not follow the boundary of the point cloud and replaces the original points with new points laying on a reconstructed continuous surface, satisfying the Poisson's differential equation. The size of the triangles increases outside the region containing the original points. The IBT algorithm produces the expected result, generating a meshed surface stripe, with no redundant or unwanted triangles. The new approach appears to be extremely advantageous over other methods, to generate $\mathrm{C}$-scan representations for shapes with concave regions. Moreover, the IBT method gives a clean and fault-free $\mathrm{C}$-scan presentation even on highly complex surface geometries.

\section{CONCLUSIONS}

New data visualization and data analysis tools, suitable to screen the NDT information obtained through high speed inspection systems, are urgently required to decrease the time needed for data analysis. The novel Index Based Triangulation (IBT) method was introduced and described. The approach is applicable to ultrasonic data gathered in a structured and orderly fashion such as that obtained from ultrasonic phased array probes. The method produces 3D C-scans, presented as coloured tessellated surfaces using an indexing method. The approach works efficiently on challenging geometries incorporating concave and convex regions. Qualitative and quantitative results have been presented. The IBT C-scan generation resulted up to 500 times faster than other techniques.

\section{REFERENCES}

[1] C. Mineo, S. G. Pierce, B. Wright, P. I. Nicholson, and I. Cooper, "Robotic path-planning for non-destructive testing of complex shaped surfaces," in QNDE Conference, Boise, Idaho, USA, 2014.

[2] T. Sattar, "Robotic non-destructive testing," Industrial Robot: An International Journal, vol. 37, 2010.

[3] J. T. Stetson and W. D. Odorico, "Robotic inspection of fiber reinforced aerospace composites using phased array UT," presented at the 40th Annual Review of Progress in Quantitative NDE, Baltimore, Maryland, 2013.

[4] E. Cuevas, S. Hernandez, and E. Cabellos, "Robot-Based Solutions for NDT Inspections: Integration of Laser Ultrasonics and Air Coupled Ultrasounds for Aeronautical Components," in 25th ASNT Research Symposium, 2016, pp. 39-46.

[5] W. A. Levinson, "Control Charts Control Multiple Attributes," Quality, vol. 43, p. 40, 2004.

[6] C. Mineo, C. MacLeod, M. Morozov, S. G. Pierce, T. Lardner, R. Summan, et al., "Fast ultrasonic phased array inspection of complex geometries delivered through robotic manipulators and high speed data acquisition instrumentation," in Ultrasonics Symposium (IUS), 2016 IEEE International, 2016, pp. 1-4.

[7] C. Mineo, S. Pierce, B. Wright, I. Cooper, and P. Nicholson, "PAUT inspection of complex-shaped composite materials through six DOFs robotic manipulators," Insight-Non-Destructive Testing and Condition Monitoring, vol. 57, pp. 161-166, 2015.

[8] Y. Drew, "A closer look at GPUs," Communications of the ACM, vol. $51,2008$.

[9] B. Delaunay, "Sur la sphere vide," Izv. Akad. Nauk SSSR, Otdelenie Matematicheskii i Estestvennyka Nauk, vol. 7, pp. 1-2, 1934.

[10] M. Kazhdan and H. Hoppe, "Screened poisson surface reconstruction," ACM Transactions on Graphics (TOG), vol. 32, p. 29, 2013. 